\title{
ON A THEOREM OF FROBENIUS
}

\section{KÄTE FENCHEL}

Let $G$ be a finite group of order $|G|$. By Frobenius' famous theorem [2] the number of solutions of the equation $x^{n}=1, x \in G, n|| G \mid$, is an integral multiplum of $n$. Frobenius conjectured that

$$
L_{n}=L_{n}(G)=\left\{x \in G \mid x^{n}=1\right\}
$$

is a subgroup (of course characteristic), if $\left|L_{n}\right|=n$. In the following we consider the case $(n,|G| / n) \neq 1$. (This condition does not play a rôle in Lemma $1-$ Corollary 4).

Notations. $P_{\gamma}$ is a subgroup of order $p^{\gamma}, \mathfrak{N}_{G}\left(P_{\gamma}\right)=N_{\gamma},|G| /\left|N_{\gamma}\right|=h_{\gamma}, \mathfrak{C}_{G}\left(P_{\gamma}\right)$ $=C_{\gamma}, P_{\alpha} \in \operatorname{Syl}_{p}(G), m_{\gamma}=\left(\left|N_{\gamma}\right|, n\right), c_{\gamma}=\left(\left|C_{\gamma}\right|, \dot{n}\right)$.

Let $n=n^{\prime} p^{\beta},\left(n^{\prime}, p\right)=1,\left|L_{n}\right|=n s_{\beta},\left|L_{n / p}\right|=(n / p) s_{\beta-1}$ with integral $s_{\beta}, s_{\beta-1}$. Then

$$
L_{n}=L_{n / p} \cup L^{\prime} \text {, }
$$

where

$$
L^{\prime}=\left\{z=x y|| x \mid=p^{\beta}, y \in \mathbb{C}_{G}(x) \cap L_{n^{\prime}}\right\} .
$$

Since $L_{n / p} \cap L^{\prime}=\varnothing$, we get

$$
n s_{\beta}=\frac{n}{p} s_{\beta-1}+\left|L^{\prime}\right| .
$$

Notice that for a subgroup $H \subset G, H \cap L_{n}(G)=L_{(n,|H|)}(H)$ so that

$$
L^{\prime}=\left\{z=x y|| x \mid=p^{\beta}, y \in L_{c_{\beta}^{\prime}}\left(C_{\beta}\right)\right\},
$$

where

$$
c_{\beta}=c_{\beta}^{\prime} p^{\beta}, \quad\left(c_{\beta}^{\prime}, p\right)=1
$$

Lemma 1. $\varphi\left(p^{\beta}\right)$ divides $\left|L^{\prime}\right|$. 
The proof is immediate, since by (3) every cyclic subgroup $\langle x\rangle$ of order $p^{\beta}$ contributes $\varphi\left(p^{\beta}\right)$ elements to $L^{\prime}$.

Hence we have

$$
L^{\prime}=\varphi\left(p^{\beta}\right)\left|\left\{\langle x\rangle y \mid y \in L_{c_{\beta}^{\prime}}\left(C_{\beta}\right)\right\}\right|,
$$

where $\langle x\rangle$ runs through the cyclic subgroups of order $p^{\beta}$ in $G$. In the case $\left|L_{n}\right|$ $=n$, that is, $s_{\beta}=1$ we get by (2) and (4)

$$
\frac{n^{\prime}\left(p-s_{\beta-1}\right)}{p-1}=\left|\left\{\langle x\rangle y \mid y \in L_{c^{\prime}}\left(C_{\beta}\right)\right\}\right|
$$

with the same range of $\langle x\rangle$ as in (4). The right hand side can be interpreted in two ways:

(A) It equals the number of cosets of the form $\langle x\rangle y$, where $|x|=p^{\beta}$, $y \in L_{c_{\beta}^{\prime}}\left(C_{\beta}\right)$.

(B) It equals the number of cosets of the form $\langle x\rangle y$, where $y \in L_{n^{\prime}}(G)$, $\langle x\rangle \subset \mathfrak{C}_{G}(y),|x|=p^{\beta}$.

Frobenius' conjecture is valid for $n=p^{\beta}$ and for solvable groups [4, Theorem $9 ; 4,1]$. Let $|G|+n$ be minimal so that the conjecture is false. It follows immediately that $n \neq|G|$ and that $L_{n}$ is not contained in a proper subgroup of $G$. We assume this minimal condition throughout the following.

Lemma 2. Suppose $\left|L_{n}\right|=n$, where $L_{n}$ satisfies the minimal condition. Then no normal subgroup of $G$ is contained in $L_{n}$.

Proof. Suppose $H \triangleleft G, H \subset L_{n}$. Let $n=n_{1} d,|H|=h_{1} d,\left(n_{1}, h_{1}\right)=1$ so that $n_{1}|| G / H \mid$. By Frobenius' theorem $\bar{x}^{n_{1}}=\overline{1}$ has $n_{1} t$ solutions in $\bar{G}=G / H$ with integral $t$. If $x \rightarrow \bar{x}$ in the natural homomorphism $G \rightarrow \bar{G}$, then $x^{n_{1}} \in H$. For $g \in H \subset L_{n}$ we have $g^{n}=g^{|H|}=1$, hence $g^{d}=1$ and consequently $x^{n_{1} d}=x^{n}=1$ for $n_{1} t|H|$ elements of $G$. Since $\left|L_{n}\right|=n, n_{1} t|H| \leqq n$. Thus $|H|=d$ and $t=1$. By the minimal condition $L_{n_{1}}(\bar{G})=\bar{K}$ is a group of order $n_{1}$ and the inverse image $K$ of $K$ in $G$ has order $n_{1} d=n$. Then $K=L_{n}$ is a group, a contradiction.

Lemma 3. Let $\left|L_{n}\right|=n, n=n^{\prime} p^{\beta},\left(n^{\prime}, p\right)=1$ where $L_{n}$ satisfies the minimal condition. If $\left(p-1, n^{\prime}\right)=1, L_{n}$ contains the Sylow p-subgroups of $G$.

Proof. Let $P_{\alpha} \in \operatorname{Syl}_{p}(G),\left|P_{\alpha}\right|=p^{\alpha}$. Suppose $P_{\alpha} \notin L_{n}$. Then $P_{\alpha}$ must contain an element of order $p^{\beta+1}$, hence also an element of order $p^{\beta}$ so that $\left|L^{\prime}\right|>0$. If $\left(p-1, n^{\prime}\right)=1,(5)$ implies $s_{\beta-1}=1$, hence $\left|L_{n / p}\right|=n / p$. By the minimal condition $L_{n / p}$ is a normal subgroup of $G$, and it is contained in $L_{n}$, which contradicts Lemma 2. 
Corollary 4. If $\left|L_{n}\right|=n$, where $L_{n}$ satisfies the minimal condition, the Sylow subgroups belonging to the minimal prime divisor of $n$ are contained in $L_{n}$.

Proof. For odd $p$, this is a direct consequence of Lemma 3. For $p=2$ and Syl $_{2}$ subgroups $P_{\alpha} \notin L_{n}$, we have $L_{n / 2}=\frac{1}{2} n s_{\beta-1}$ and $L_{n / 2} \pm L_{n}$ so that $s_{\beta-1}<2$. Hence $s_{\beta-1}=1$ which again contradicts Lemma 2 .

THEOREM 5. Let again $L_{n}$ satisfy the minimal condition. Suppose $(n,|G| / n) \neq 1$, $p \mid(n,|G| / n)$. Then the Sylow p-subgroups of $G$ are cyclic.

Proof. Let $n=p^{\beta} n^{\prime},\left(n^{\prime}, p\right)=1, P_{\alpha} \in \operatorname{Syl}_{p}(G), p^{\alpha}>p^{\beta}$. Suppose exponent $P_{\alpha}$ $\leqq p^{\beta}$. Then $P_{\alpha} \subset L_{n}$. But $n p|| G \mid$ by $p^{\alpha}>p^{\beta}$ so that $\left|L_{n p}\right|=n p s_{\beta+1}>n, s_{\beta+1}$ integral, while $\left|L_{n p}\right|=\left|L_{n}\right|=n$ for $P_{\alpha} \subset L_{n}$. Hence exponent $P_{\alpha}>p^{\beta}$, and there exists an element of order $p^{\beta+1}$ in $G$. Similarly to (5), we obtain

$$
\frac{n^{\prime}\left(p s_{\beta+1}-1\right)}{p-1}=\left|\left\{\langle u\rangle y|| u \mid=p^{\beta+1}, y \in L_{c_{\beta+1}^{\prime}}\left(C_{\beta+1}\right)\right\}\right|
$$

where $c_{\beta+1}=c_{\beta+1}^{\prime} p^{\beta}=\left(\left|C_{\beta+1}\right|, n\right) ;\left(c_{\beta+1}^{\prime}, p\right)=1$. Using interpretation (B) and observing that the number of cyclic subgroups of order $p^{\beta+1}$ in $\mathfrak{C}_{G}(y)$ is constant for the elements in the class $(y)$ of conjugates to $y$, we get

$$
\frac{n^{\prime}\left(p s_{\beta+1}-1\right)}{p-1}=\sum_{i} h_{i} A\left(y_{i}\right)
$$

where $y_{i} \in L_{n^{\prime}}$ is a representative of its class $\left(y_{i}\right), h_{i}=\left|\left(y_{i}\right)\right|$, and $A\left(y_{i}\right)$ the number of cyclic subgroups of order $p^{\beta+1}$ in $\mathfrak{C}_{G}\left(y_{i}\right)$. Since the left hand side of (7) is coprime with $p$, there exists an $i$ such that $\left(h_{i} A\left(y_{i}\right), p\right)=1$. It follows from $\left(h_{i}, p\right)$ $=1$ that $\mathfrak{C}_{G}\left(y_{i}\right)$ contains a Sylow $p$-subgroup $P_{\alpha}$ of $G$. By a result of P. Hall [5], the number of cyclic subgroups of order $p^{\beta+1}$ in a group $\mathfrak{C}_{G}\left(y_{i}\right)$ with Sylow $p$-subgroup $P_{\alpha}$ is for $\beta>0$ a multiplum of $p^{k_{\beta}-\beta}$. Here, according to P. Hall:

“(i) If $P_{\alpha}$ is regular, we may take $k_{\beta}=\varrho_{\beta}$ where $\varrho_{\beta}$ is the order of $\Omega_{\beta}\left(P_{\alpha}\right)$.

(ii) If $P_{\alpha}$ is irregular and the elements of order less than or equal to $p^{\beta}$ in $P_{\alpha}$ form a subgroup $\Omega_{\beta}\left(P_{\alpha}\right)$, we may take $k_{\beta}=\min \left\{\varrho_{\beta}, \beta(p-1)\right\}$.

(iii) If $P_{\alpha}$ is irregular and the elements of order less than or equal to $p^{\beta}$ in $P_{\alpha}$ do not form a subgroup, we may take $k_{\beta}=\beta(p-1)$."

Since $\left(A\left(y_{i}\right), p\right)=1$, we must have $p^{k_{\beta}-\beta}=1$, hence $k_{\beta}=\beta$. By Corollary $4, p \neq 2$, thus $\beta(p-1)>\beta$. Therefore only (i) and (ii) are possible, and we obtain $\left|\Omega_{\beta}\left(P_{\alpha}\right)\right|$ $=p^{\beta}$. Since there exists an element of order $p^{\beta}$ in $P_{\alpha}, \Omega_{\beta}\left(P_{\alpha}\right)$ is the only subgroup of that order in $P_{\alpha}$, and $P_{\alpha}$ is cyclic, since $p$ is odd. 
Remark. Theorem 5 was proved by Richard Zemlin [7] in a different way in an unpublished $\mathrm{PhD}$ dissertation, Ohio State University 1954. He also proved that, under the condition $(n,|G| / n) \neq 1, G$ must be simple, if the conjecture is false. We do not use this last fact in the following.

Corollary 6. Let $H$ be a subgroup of $G, H$ in $L_{n}$ (satisfying the minimal condition). Then $|H| \mid n$.

Proof. Let $|H|=h_{1} q^{\delta},\left(h_{1}, q\right)=1, Q_{\alpha} \in \operatorname{Syl}_{q}(G)$. Either $(q,|G| / n) \neq 1$, so that $Q_{\alpha}$ is cyclic by Theorem 5, and there exists an element of order $q^{\delta}$ in $H \subset L_{n}$. Hence $q^{\delta} \mid n$. Or $(q,|G| / n)=1$. Then certainly $q^{\delta} \mid n$.

Let now $p$ be the minimal prime divisor of $(n,|G| / n)$. By Corollary $4, p \neq 2$, and by Theorem 5, the Sylow $p$-subgroups $P_{\alpha}$ are cyclic. Hence all subgroups $P_{\beta}=\langle x\rangle$ of order $p^{\beta}$ are conjugate and $\left|L_{c_{\beta}^{\prime}}\left(C_{\beta}\right)\right|$ is constant (interpretation $(A)$ ). The number of cyclic subgroups of order $p^{\beta}$ in $G$ is $|G| /\left|N_{\beta}\right|=h_{\beta}$, where $P_{\beta}$ is a fixed $p^{\beta}$-subgroup in $L_{n}$. By (5)

$$
\frac{n^{\prime}\left(p-s_{\beta-1}\right)}{p-1}=h_{\beta}\left|L_{c^{\prime}}\left(C_{\beta}\right)\right|, \quad p>s_{\beta-1}>1
$$

Similarly to (8) we get with respect to $N_{\beta}$

$$
\frac{m_{\beta}^{\prime}\left(p t_{\beta}-t_{\beta-1}\right)}{p-1}=\left|L_{c_{\beta}^{\prime}}\left(C_{\beta}\right)\right|=c_{\beta}^{\prime} T_{\beta},
$$

where $\left|L_{m_{\beta}}\left(N_{\beta}\right)\right|=m_{\beta} t_{\beta},\left|L_{m_{\beta} / p}\left(N_{\beta}\right)\right|=t_{\beta-1} m_{\beta} / p, m_{\beta}=m_{\beta}^{\prime} p^{\beta},\left(m_{\beta}^{\prime}, p\right)=1, t_{\beta}, t_{\beta-1}$ and $T_{\beta}$ integral $\geqq 1$ and

$$
\left|L_{c_{\beta}}\right|=c_{\beta} T_{\beta}=p^{\beta} c_{\beta}^{\prime} T_{\beta}=p^{\beta}\left|L_{c_{\beta}^{\prime}}\left(C_{\beta}\right)\right|,
$$

since $\dot{L_{c_{\beta}}}\left(C_{\beta}\right)=P_{\beta} \times L_{c_{\beta}^{\prime}}\left(P_{\beta}\right)$.

LeMma 7. $p t_{\beta}-t_{\beta-1}$ divides $p-s_{\beta-1}$.

Proof. By (8) and (9)

$$
n^{\prime}\left(p-s_{\beta-1}\right)=m_{\beta}^{\prime} h_{\beta}\left(p t_{\beta}-t_{\beta-1}\right)
$$

or

$$
\left|N_{\beta}\right|=\frac{|G|}{n} m_{\beta} \frac{\left(p t_{\beta}-t_{\beta-1}\right)}{p-s_{\beta-1}} .
$$

Using $\left(\left|N_{\beta}\right|, n\right)=m_{\beta}$ as well as (10) and (11), we get 


$$
1=\left(\left|N_{\beta}\right| / m_{\beta}, n / m_{\beta}\right)=\left(\frac{|G|}{n} \frac{p t_{\beta}-t_{\beta-1}}{p-s_{\beta-1}}, \frac{h_{\beta}\left(p t_{\beta}-t_{\beta-1}\right.}{p-s_{\beta-1}}\right),
$$

which proves the Lemma.

Set $\left|L_{m_{\beta} p}\left(N_{\beta}\right)\right|=m_{\beta} p t_{\beta+1}, t_{\beta+1}$ integer, $t_{\beta+1} \geqq 1$. Then, corresponding to (8) and (9), we have (see (6)) for $c_{\beta+1}=c_{\beta+1}^{\prime} p^{\beta}=\left(\left|C_{\beta+1}\right|, n\right), \quad\left(c_{\beta+1}^{\prime}, p\right)=1$ and $\left|L_{c_{\beta+1}^{\prime}}\left(C_{\beta+1}\right)\right|=c_{\beta+1}^{\prime} T_{\beta+1}, T_{\beta+1} \geqq 1$ integral,

$$
\begin{gathered}
\frac{n^{\prime}\left(p s_{\beta+1}-1\right)}{p-1}=\frac{|G|}{\left|N_{\beta+1}\right|}\left|L_{c_{\beta+1}^{\prime}}\left(C_{\beta+1}\right)\right|=h_{\beta+1} c_{\beta+1}^{\prime} T_{\beta+1} \text { in } G \\
\frac{m_{\beta}^{\prime}\left(p t_{\beta+1}-t_{\beta}\right)}{p-1}=\frac{\left|N_{\beta}\right|}{\left|N_{\beta+1}\right|}\left|L_{c_{\beta+1}^{\prime}}\left(C_{\beta+1}\right)\right|=\frac{h_{\beta+1}}{h_{\beta}} c_{\beta+1}^{\prime} T_{\beta+1} \text { in } N_{\beta} .
\end{gathered}
$$

Combining (12) and (13), we get

$$
n^{\prime}\left(p s_{\beta+1}-1\right)=h_{\beta} m_{\beta}^{\prime}\left(p t_{\beta+1}-t_{\beta}\right),
$$

and by (10)

$$
\frac{p s_{\beta+1}-1}{p t_{\beta+1}-t_{\beta}}=\frac{p-s_{\beta-1}}{p t_{\beta}-t_{\beta-1}}
$$

Hence

$$
t_{\beta-1} \equiv s_{\beta-1} t_{\beta} \quad(\text { modulo } p) .
$$

Let now $P_{\alpha} \subset N_{\beta}$ be fixed. Since $P_{\alpha}$ is cyclic and $p \neq 2, N_{\alpha} / C_{\alpha}$ is a cyclic group of an order $r \mid p-1$ so that

$$
N_{\alpha}=C_{\alpha} \cup C_{\alpha} y \cup \ldots \cup C_{\alpha} y^{r-1}, \quad y^{r} \in C_{\alpha} .
$$

By Herzog [6]

$$
\left|N_{\alpha} / C_{\alpha}\right|=\left|N_{\beta} / C_{\beta}\right|
$$

and the coset representatives of $N_{\alpha}$ modulo $C_{\alpha}$ can also be taken as coset representatives of $N_{\beta}$ modulo $C_{\beta}$ so that also

$$
N_{\beta}=C_{\beta} \cup C_{\beta} y \cup \ldots \cup C_{\beta} y^{r-1} .
$$

Further $y^{b}$ has no fixed point on $P_{\alpha}, b=1, \ldots, r-1$.

Since $p$ is the minimal divisor of $(n,|G| / n)$ and $r / p-1$, no prime divisor $q$ of $r$ divides $(n,|G| / n)$. Consequently the Sylow $q$-subgroups of $G$ are either contained in $L_{n}$ or in $L_{|G| / n}$. Thus for $r=r_{1} r_{2}, r_{1}=(n, r)$, we have $r_{2}=(|G| / n, r)$ and $\left(r_{1}, r_{2}\right)=1$. 
LEMMA 8. $m_{\beta} / c_{\beta}=r_{1}$.

Proof. By taking the greatest common divisor with $m_{\beta}$ on both sides of $\left|N_{\beta}\right|$ $=\left|C_{\beta}\right| r$ we get

$$
m_{\beta}=c_{\beta}\left(m_{\beta} / c_{\beta}, r\right)=c_{\beta}\left(m_{\beta} / c_{\beta}, r_{1}\right),
$$

since $\left(m_{\beta} / c_{\beta}, r_{2}\right)=1$, such that $m_{\beta} / c_{\beta} \mid r_{1}$.

On the other hand, let $q^{\delta}$ be the highest power of the prime $q$ dividing $r_{1}$, and let $Q_{\alpha} \in \operatorname{Syl}_{q}\left(N_{\beta}\right), Q_{\gamma}=Q_{\alpha} \cap C_{\beta} \in \operatorname{Syl}_{q}\left(C_{\beta}\right)$. Then $q^{\delta}=\left|Q_{\alpha}\right| /\left|Q_{\gamma}\right|$. Let $Q$ be a Sylow $q$-subgroup of $G$ containing $Q_{\alpha}$. By the remark above, $Q \subset L_{n}$, since $(q,|G| / n)=1$ so that $Q_{\alpha} \subset L_{n} \cap N_{\beta}=L_{m_{\beta}}$. Then

$$
\left|Q_{\alpha}\right| \mid\left(n,\left|N_{\beta}\right|\right), \quad\left(n,\left|N_{\beta}\right|\right)=m_{\beta},
$$

by Corollary 6 . In the same way we get $\left|Q_{\gamma}\right| \mid c_{\beta}$, and hence $\left|Q_{\alpha}\right| /\left|Q_{\gamma}\right|=q^{\delta}$ divides $m_{\beta} / c_{\beta}$. Since this is valid for all prime divisors of $r_{1}$, the Lemma is proved.

THEOREM 9. Suppose $\left|L_{n}\right|=n|| G \mid,(n,|G| / n) \neq 1$. Then the assumption that the Frobenius conjecture is valid for all pairs $\left\{n_{1},\left|G_{1}\right|\right\}$ with $n_{1}+\left|G_{1}\right|<n+|G|$ and is false for the pair $\{n,|G|\}$ leads to a contradiction.

Proof. (i) Suppose first $m_{\beta} / c_{\beta}=m_{\beta}^{\prime} / c_{\beta}^{\prime}=r_{1}=1$. Then we get by (9) and Lemma 8

$$
\frac{r_{1}\left(p t_{\beta}-t_{\beta-1}\right)}{p-1}=\frac{p t_{\beta}-t_{\beta-1}}{p-1}=T_{\beta},
$$

where $T_{\beta}$ is an integer $\geqq 1$. This is impossible, since by Lemma $7, p t_{\beta}-t_{\beta-1} \leqq$ $p-s_{\beta-1}$ which is less than $p-1$, if the Frobenius conjecture is false for the pair $\{n,|G|\}$. For, by the same argument as in Lemma $3, p-s_{\beta-1}=p-1$ gives a contradiction to Lemma 2.

(ii) Hence we must assume $r_{1} \neq 1$. By (17)

$$
L_{m_{\beta}}=N_{\beta} \cap L_{m_{\beta}}=\left(C_{\beta} \cap L_{m_{\beta}}\right) \cup\left(C_{\beta} y \cap L_{m_{\beta}}\right) \cup \ldots \cup\left(C_{\beta} y^{r-1} \cap L_{m_{\beta}}\right)
$$

such that

$$
m_{\beta} t_{\beta}=\left|L_{c_{\beta}}\right|+\left|C_{\beta} y \cap L_{m_{\beta}}\right|+\ldots+\left|C_{\beta} y^{r-1} \cap L_{m_{\beta}}\right| .
$$

Since $N_{\beta} / C_{\beta}$ is cyclic, $\left[N_{\beta}, N_{\beta}\right] \subseteq C_{\beta}$, and the cosets $C_{\beta} y^{b}$ consist of whole classes of (in $N_{\beta}$ ) conjugate elements as does $L_{m_{\beta}}$ such that also $C_{\beta} y^{b} \cap L_{m_{\beta}}$ consists of whole classes of $N_{\beta}$. The number of elements in a class $\left(x y^{b}\right), x \in C_{\beta}$, is $\left|N_{\beta}: \mathfrak{C}_{N_{\beta}}\left(x y^{b}\right)\right|$. Now no subgroup of $p$-power order can be centralized by $x y^{b}$. For if this were the case, $x y^{b}$ would also centralize $\Omega_{1}\left(P_{\gamma}\right)$ which equals $\Omega_{1}\left(P_{\beta}\right)$ 
for all $P_{\gamma} \subset N_{\beta}$. But $x y^{b}$ is a $p^{\prime}$-element, since all $p$-irregular elements of $L_{m_{\beta}}$ are contained in $L_{c_{\beta}}$. Since $x y^{b}$ normalizes $P_{\beta}$, it would consequently centralize $P_{\beta}$ $[3$, Theorem $5 ; 3,10]$ which is not the case for $b<r$. Hence $\mathfrak{C}_{N_{\beta}}\left(x y^{b}\right)$ contains no p-element so that

$$
\left(\left|\mathfrak{C}_{N_{\beta}}\left(x y^{b}\right)\right|, p\right)=1 \quad \text { and } \quad\left|N_{\beta}: \mathfrak{C}_{N_{\beta}}\left(x y^{b}\right)\right| \equiv 0\left(\operatorname{modulo} p^{\alpha}\right) .
$$

Since this is valid for all classes in $C_{\beta} y^{b} \cap L_{m_{\beta}}$

$$
\left|C_{\beta} y^{b} \cap L_{m_{\beta}}\right| \equiv 0\left(\text { modulo } p^{\alpha}\right) \quad \text { for all } b=1, \ldots, r-1 \text {. }
$$

(For $b \neq 0$ (modulo $r_{2}$ ) even $C_{\beta} y^{b} \cap L_{m_{\beta}}=\varnothing$, as is easily seen). With

$$
\left|L_{c_{\beta}}\right|=c_{\beta} T_{\beta}=\frac{m_{\beta}\left(p t_{\beta}-t_{\beta-1}\right)}{p-1}
$$

we then have by (19)

$$
m_{\beta} t_{\beta} \equiv \frac{m_{\beta}\left(p t_{\beta}-t_{\beta-1}\right)}{p-1} \quad\left(\operatorname{modulo} p^{\alpha}\right)
$$

or

$$
m_{\beta}^{\prime} t_{\beta} \equiv \frac{m_{\beta}^{\prime}\left(p t_{\beta}-t_{\beta-1}\right)}{p-1} \quad\left(\operatorname{modulo} p^{\alpha-\beta}\right)
$$

where $\alpha-\beta \geqq 1$

which implies

$$
t_{\beta-1} \equiv t_{\beta} \quad\left(\operatorname{modulo} p^{\alpha-\beta}\right),
$$

since $\left(m_{\beta}^{\prime}, p\right)=1$. By $(16)$

$$
t_{\beta-1} \equiv s_{\beta-1} t_{\beta} \quad(\text { modulo } p) .
$$

Combining (21) and (22), we get

$$
t_{\beta-1}\left(s_{\beta-1}-1\right) \equiv 0 \quad(\text { modulo } p) .
$$

Now $\quad s_{\beta-1}<p$, because $\left|L_{n / p}\right|=(n / p) s_{\beta-1}<\left|L_{n}\right|=n$ so that $s_{\beta-1}-1 \equiv 0$ (modulo $p$ ) implies $s_{\beta-1}=1$ which contradicts the minimal condition, as we saw above. Hence $t_{\beta-1} \equiv t_{\beta} \equiv 0$ (modulo $p$ ) by (21) and thus

$$
T_{\beta}=\frac{r_{1}\left(p t_{\beta}-t_{\beta-1}\right)}{p-1} \equiv 0 \quad(\text { modulo } p) .
$$

But $T_{\beta}<r_{1} \leqq p-1$, since $p t_{\beta}-t_{\beta-1}<p-1$ so that we would have $T_{\beta}=0$ which is absurd. Hence the Frobenius conjecture is proved in the case $(n,|G| / n) \neq 1$. 
AdDENDUM. If $\left|L_{n}\right|=n=p^{\beta} n^{\prime}$ and $p \mid(n,|G| / n)$, all elements of $L_{n}$, that normalize $P_{\beta}$, centralize $P_{\beta}$, i.e. $r_{1}=1$.

Proof. By R. Brauer's definition [1], $N_{\beta}$ is a group of metacyclic type which means the following: $N_{\beta}$ has a cyclic Sylow $p$-subgroup $P_{\alpha}$ and a normal subgroup $K\left(=O_{p^{\prime}}\left(N_{\beta}\right)\right)$ of index $p^{\alpha} r$ such that $\bar{N}_{\beta}=N_{\beta} / K$ is a metacyclic group of order $p^{\alpha} r$ defined by

$$
\bar{N}_{\beta}=\left\langle\bar{x}_{\alpha}, \bar{y}, \bar{x}_{\alpha}^{p^{\alpha}}=\overline{1}, \bar{y}^{r}=\overline{1}, \bar{y}^{-1} \bar{x}_{\alpha} \bar{y}=\bar{x}_{\alpha}^{j}\right\rangle,
$$

where $j$ belongs to exponent $r$ modulo $p^{\alpha}$. Here $O_{p^{\prime}}\left(N_{\beta}\right) \subset C_{\beta}, N_{\beta} / C_{\beta}$ is cyclic of order $r$ and $C_{\beta}=O_{p^{\prime}}\left(N_{\beta}\right) P_{\alpha}$ by Burnside's theorem, since $P_{\alpha} \subset C_{\beta}$ and $P_{\alpha}$ is contained in the center of its normalizer $\mathfrak{N}_{C_{\beta}}\left(P_{\alpha}\right)=N_{\alpha} \cap C_{\beta}$ which equals $C_{\alpha}$ by Herzog [6].

By Theorem $9, L_{n}$ is a group. Then also $L_{n} \cap N_{\beta}$ and $\bar{L}_{m_{\beta}}$ $=L_{m_{\beta}} O_{p^{\prime}}\left(N_{\beta}\right) / O_{p^{\prime}}\left(N_{\beta}\right)$ are groups. $\bar{g} \in \bar{L}_{m_{\beta}}$ satisfies the conditions $\bar{g}^{m_{\beta}}=\overline{1}$ and $\bar{g}^{p^{\alpha} r}=\overline{1}$, hence

$$
\bar{L}_{m_{\beta}}=\left\{\bar{g} \in \bar{N}_{\beta} \mid \bar{g}^{r_{1} p^{\beta}}=\overline{1}\right\} .
$$

If $r_{1} \neq 1, \bar{L}_{m_{\beta}}$ contains an element $\bar{y}_{1}$ of an order dividing $r_{1}$, and consequently $\bar{L}_{m_{\beta}}$ contains the whole class $\left(\bar{y}_{1}\right)$ of conjugate elements in $N_{\beta}$ which consists of all elements $\bar{x}_{\alpha}^{b} \bar{y}_{1}, b=0, \ldots, p^{\alpha}-1$. Since $\bar{L}_{m_{\beta}}$ is a group and $\bar{y}_{1}$ and $\bar{x}_{\alpha} \bar{y}_{1}$ are contained in $\bar{L}_{m_{\beta}}$, also $\bar{x}_{\alpha}$ would be contained in $\bar{L}_{m_{\beta}}$ which is not the case, since $\left|\bar{x}_{\alpha}\right|=p^{\alpha}>p^{\beta}$. Hence $r_{1}=1$.

\section{REFERENCES}

1. R. Brauer, On finite groups with cyclic subgroups. I. J. Algebra 40 (1976), 556-584.

2. F. G. Frobenius, Uber einen Fundamentalsatz der Gruppentheorie, I., II. Gesammelte Abhandlungen, III, 330-334, 394-403. Springer-Verlag, Berlin - Heidelberg - New York, 1968.

3. D. Gorenstein, Finite groups, Harper and Row, New York - Evanston - London, 1968.

4. M. Hall, Jr., The theory of groups, Macmillan, New York, 1959.

5. P. Hall, On a theorem of Frobenius, Proc. London Math. Soc. (2) 40 (1936), 468-501.

6. M. Herzog, On finite groups with cyclic Sylow subgroups, Israel J. Math. 6 (1968), 206-216.

7. R. A. Zemlin, On a conjecture arising from a theorem of Frobenius, Unpublished $\mathrm{PhD}$ Dissertation, Ohio State University, 1954. 